\title{
Anorectal swabs as a marker of male-to-male sexual exposure in STI surveillance systems
}

\author{
F. H. AMPT ${ }^{1,2}$, C. EL HAYEK ${ }^{1 *}$, P. A. AGIUS ${ }^{1,2,3}$, A. L. BOWRING ${ }^{1}$, \\ N. BARTNIK ${ }^{1}$, C. VAN GEMERT ${ }^{1,2}$, C. K. FAIRLEY ${ }^{4,5}$, E. P. F. $\mathrm{CHOW}^{4,5}$, \\ C. S. BRADSHAW ${ }^{4,5}$, N. STEPHENS ${ }^{6}$, M. S. C. LIM ${ }^{1,2}$ AND M. E. HELLARD ${ }^{1,2}$ \\ ${ }^{1}$ Burnet Institute, Melbourne, Victoria, Australia \\ ${ }^{2}$ Department of Epidemiology and Preventive Medicine, Monash University, Melbourne, Victoria, Australia \\ ${ }^{3}$ Judith Lumley Centre, La Trobe University, Melbourne, Victoria, Australia \\ ${ }^{4}$ Melbourne Sexual Health Centre, Alfred Health, Carlton, Victoria \\ ${ }^{5}$ Central Clinical School, Faculty of Medicine, Nursing and Health Sciences, Monash University, Melbourne, \\ Victoria, Australia \\ ${ }^{6}$ Department of Health Victoria, Melbourne, Victoria, Australia
}

Received 22 December 2016; Final revision 29 March 2017; Accepted 19 April 2017; first published online 22 May 2017

\section{SUMMARY}

Identification of priority populations such as men who have sex with men (MSM) is important in surveillance systems to monitor trends of sexually transmitted infections (STIs). We explored using routinely collected non-behavioural data as a means to establish MSM status in surveillance by assessing anorectal swab as a marker of male-to-male sexual exposure. We used chlamydia testing data from a sexual health clinic, 2007-2012. Men reporting any male sexual partner(s) in the previous 12 months were considered MSM. The dataset was split into development and validation samples to develop a univariate predictive model and assess the model fit. The dataset included 30358 individual men and 48554 episodes of STI testing; $45 \%$ were among reported MSM and an anorectal swab was performed in $40 \%$ of testing episodes. Anorectal swabbing had good diagnostic performance as a marker for MSM status (sensitivity $=87 \%$, specificity $=99 \%$, positive predictive value $=98 \cdot 6 \%$, negative predictive value $=90 \cdot 3 \%$ ). The model showed good fit against the internal validation sample (area under the curve $=0 \cdot 93$ ). Anorectal swabs are a valid marker of MSM behaviour in surveillance data from sexual health clinics, and they are likely to be particularly useful for monitoring STI trends among MSM with higher risk behaviour.

Key words: Chlamydia, men who have sex with men - MSM, sexually transmitted infections, surveillance.

\section{INTRODUCTION}

Surveillance of sexually transmitted infections (STIs) allows trends in STI epidemiology to be identified

\footnotetext{
* Author for correspondence: C. El Hayek, Burnet Institute, 85 Commercial Rd, Melbourne, Victoria 3004, Australia. (Email: carol.el-hayek@burnet.edu.au)
}

and the impacts of interventions and screening programs to be evaluated. Identification of key populations such as men who have sex with men (MSM), young people and Aboriginal and Torres Strait Islanders in surveillance systems is important to be able to monitor trends and epidemics of STIs in priority populations for STI control [1-3]. 
In addition to passive surveillance, in which positive results are reported to a public health authority, some jurisdictions operate active surveillance systems. These have the advantage of capturing negative as well as positive testing data, and may target specific high-risk populations from sentinel sites, such as sexual health services. Sentinel surveillance systems have also been established to capture testing data from public and private laboratories (for example, the Australian Collaboration for Coordinated Enhanced Sentinel Surveillance of Blood Borne Viruses and Sexually Transmitted Infections (ACCESS), a multijurisdictional Australian system [4]). Laboratory-based surveillance can be used to estimate the prevalence of STIs (by monitoring the proportion of tests that are positive), as well as incidence and reinfection rates, among large populations from varied clinical settings. However, it is difficult to identify key populations within these datasets, due to the limited recording of behavioural information (for example, gender of sexual partners) in laboratory records and by clinicians on pathology request forms. Therefore, other means of identifying at-risk populations such as MSM are required in systems where sexual exposure data are not available.

Australian and international guidelines recommend that asymptomatic STI screening be conducted among MSM at least annually, and at multiple anatomical sites (urethral, pharyngeal and anorectal) [5-7]. As specimen site is captured by laboratory testing data, and it is unlikely that men would have an anorectal swab if not engaging in receptive anal intercourse, particularly since anorectal STIs are largely asymptomatic $[8,9]$, swab for anorectal chlamydia may be a valid marker of male-to-male sexual exposure.

Therefore, the objectives of this study were to determine the predictive value of anorectal swab site for male-to-male sexual exposure and whether having an anorectal swab can be used as a proxy to identify MSM in STI surveillance systems.

\section{METHODS}

\section{Setting}

The model was developed and internally validated using a dataset derived from the Victorian Primary Care Network for Sentinel Surveillance on Blood Borne Viruses and STIs (VCPNSS) [10]. VPCNSS links HIV and STI testing data to sexual behavioural data through a network of selected sentinel clinical sites and laboratories, including clinics that see a majority of MSM, sexual health clinics, family planning clinics and community health clinics that see both MSM and non-MSM populations. VPCNSS allows for uniquely identified individuals to be followed over time.

This analysis was limited to Melbourne Sexual Health Centre (MSHC), which recorded sexual exposure for all patients enabling MSM to be identified within the overall patient group; high caseload MSM clinics were excluded due to small number of non-MSM for comparison, and other clinics excluded due to small number of anorectal tests and insufficient behavioural data.

\section{Study population}

Data were limited to all chlamydia tests among men taken at any anatomical site at MSHC between 2007 and 2012. This provided a dataset with sufficient power for both model development and validation. Chlamydia testing data were used exclusively because it is the only STI with a generalised epidemic in Victoria (prevalent in both MSM and non-MSM) [11]. We included laboratory test records that matched to a completed questionnaire (see below), and we excluded test records from sex workers and known HIV-positive men receiving chronic disease management at MSHC, as these groups have specific testing patterns which may have introduced selection bias.

\section{Data and definitions}

A test collection episode was defined as one or more chlamydia tests in one individual within 1 week this could include testing at multiple anatomical sites and sometimes re-testing of the same anatomical site, for example in the case of inadequate samples. An anorectal test collection was defined as any chlamydia test collection that included an anorectal swab.

Attendees at MSHC answer an electronic questionnaire on self-reported sexual behaviour, including the number and gender of their sexual partners in the previous 12 months. Men who reported having at least one male sexual partner in the 12 months prior to the test collection were considered to be MSM. Age at testing was calculated using the patient's date of birth and specimen collection date (first specimen collection within each collection episode). Additional demographic (age, country of birth) and sexual risk variables (number and gender of sex partners in 
previous 12 months, condom use during anal and vaginal sex in previous 12 months) were extracted from the questionnaire and laboratory data for assessing differences between MSM who had and had not had anorectal swabs, as detailed in Table 4.

\section{Statistical analyses}

A split-sample method was applied to the MSHC dataset to develop and internally validate a predictive model. Individual test records were randomly allocated equally to either a development or validation sample.

\section{Model development}

Generalised linear modelling (GLM) between anorectal test collection and self-report MSM status was undertaken on the development sample to quantify the diagnostic accuracy of anorectal collection in determining MSM status.

Using the development sample, we calculated the following statistics to estimate the accuracy of anorectal swab to predict MSM status at test collection episode: diagnostic odds ratio (of MSM among anorectal vs. non-anorectal test groups), sensitivity, specificity, positive and negative predictive values, and positive and negative likelihood ratios.

\section{Validation}

Predicted probabilities from GLM analyses on the development sample were then used to classify test episodes and assess the fit of the univariate model on the internal validation sample. Hosmer-Lemeshow goodness-of-fit statistic [12], area under the receiver operator curve statistic (AUC) and proportional reduction in error of classification $(\lambda \mathrm{p})$ were estimated to provide a range of fit measures. Given the $2 \times 2$ nature of the data, the AUC represents the average classification accuracy of anorectal swabbing across sensitivity and specificity; and $\lambda p$ indicates the \% reduction in error in correct classification of MSM given knowledge of anorectal swabbing.

We then assessed whether identifying MSM by anorectal swab site could be used to extrapolate results to the total MSM testing population, by comparing the demographic and behavioural characteristics and urogenital chlamydia positivity of MSM who have had anorectal swabs with those who have not. Quantile regression and $\chi^{2}$-tests of independence were used to determine whether differences were statistically significant.
In all diagnostic accuracy and sample comparative analyses, cluster robust variance estimation was used to account for the lack of independence of observations (multiple test collection episodes per individual). To account for lack of independence in model fit analyses, bootstrap standard errors were used to derive $95 \%$ confidence intervals (CI) for AUC point estimation.

All analyses were performed with Stata version 13.1 [13]. Ethics approval to undertake this study was obtained from the Alfred Health Human Ethics Committee (VPCNSS agreement, project number $213 / 05$ and project number 62/15).

\section{RESULTS}

The overall dataset included records of 30358 individual men, who underwent 48554 episodes of chlamydia testing between 2007 and 2012. Male-to-male sex in the previous 12 months was reported in $45 \%$ of test collection episodes, and an anorectal swab was performed in 40\%. MSM had on average 2.2 episodes of testing, compared with 1.3 among non-MSM $(P<0.001)$.

\section{Model development}

Of the 11002 test collections among MSM allocated to the development sample, 9597 (87\%) included anorectal swabs (Table 1). Only $141(1 \%)$ of the test collections among non-MSM included an anorectal swab (specificity 99\%; Table 2). Of the 9738 anorectal test collection episodes, $9597(98 \cdot 6 \%)$ were in men reporting sex with men.

The odds of reporting MSM was 636 (95\% CI 534 758) times higher for those who had had an anorectal swab than those who did not (Table 2).

\section{Internal validation}

Table 3 presents fit statistics for the internal validation dataset. As indicated by the AUC, goodness-of-fit statistic and estimated proportional reduction in error in classification $(\lambda \mathrm{p})$, the model fitted the internal validation sample data well.

\section{CHARACTERISTICS OF MSM WHO HAD ANORECTAL SWABS}

Compared with MSM without anorectal collection, MSM with anorectal swabs were younger, a higher proportion reported more than 10 male partners in 
Table 1. Contingency table showing anorectal swab collection by MSM status (male-to-male sex reported in last 12 months); development sample

\begin{tabular}{llll}
\hline \hline $\begin{array}{l}\text { Anorectal swab } \\
\text { done at test } \\
\text { collection episode }\end{array}$ & MSM & Not MSM & Total \\
\hline $\begin{array}{l}\text { Anorectal swab } \\
\text { Non-anorectal } \\
\text { testing }\end{array}$ & $1405(13 \%)$ & $13134(99 \%)$ & $14539(60 \%)$ \\
Total & 11002 & 13275 & 24277 \\
\hline \hline
\end{tabular}

Cell counts and column per cent.

Table 2. Diagnostic statistics and 95\% CI for assessing the diagnostic accuracy of anorectal swabbing: development sample

\begin{tabular}{ll}
\hline \hline Parameter & Estimate $(95 \% \mathrm{CI})^{*}$ \\
\hline Diagnostic odds ratio & $636(534-758)$ \\
Sensitivity & $87 \cdot 2(86 \cdot 6-87 \cdot 8)$ \\
Specificity & $98 \cdot 9(98 \cdot 7-99 \cdot 1)$ \\
Positive predictive value & $98 \cdot 6(98 \cdot 3-98 \cdot 8)$ \\
Negative predictive value & $90 \cdot 3(89 \cdot 8-90 \cdot 8)$ \\
Positive likelihood ratio & $82 \cdot 1(69 \cdot 7-96 \cdot 8)$ \\
Negative likelihood ratio & $0 \cdot 129(0 \cdot 123-0 \cdot 136)$ \\
\hline \hline
\end{tabular}

* Cluster robust standard errors specified.

Table 3. Model fit statistics for internal validation sample: AUC, Hosmer-Lemeshow goodness of fit (GOF) and proportional reduction in error of classification $(\lambda p)$

\begin{tabular}{ll}
\hline \hline Parameter & Development sample estimate $(95 \% \mathrm{CI})^{*}$ \\
\hline AUC $^{\dagger}$ & $0.93(0.93-0.93)$ \\
GOF & $\chi^{2}(2)=1.06, P=0.590$ \\
$\lambda \mathrm{p}$ & 0.86 \\
\hline \hline
\end{tabular}

* The 95\% CI based on bootstrapped standard errors. $\dagger$ AUC represents the average of anorectal swabbing sensitivity and specificity.

the previous 12 months, a higher proportion reported always using a condom during anal and vaginal sex, and a lower proportion reported male-to-female sex (Table 4). Chlamydia positivity based on urogenital test results were similar between both groups $(P=0.678)$.

\section{DISCUSSION}

Anorectal swab site is a highly predictive and valid marker of male-to-male sex within our sample from a metropolitan sexual health service. Its use as a proxy for MSM in surveillance datasets where reliable behavioural data are not available is highly acceptable, particularly for monitoring STI trends among MSM with high-risk behaviour who are more vulnerable to STI.

Despite some demographic and behavioural differences between MSM with and without an anorectal swab at test collection, there was no difference in urogenital chlamydia positivity. This suggests that using anorectal swab site for surveillance would not substantially bias estimates of urogenital chlamydia prevalence among MSM. However, differences in anorectal risk cannot be inferred due to lack of comparative biological data among MSM without anorectal testing. Although MSM with anorectal swabs reported more male sex partners than MSM without anorectal collection, they also reported more consistent condom use, perhaps indicating that men who had anorectal swabs were more aware of their risk and more likely to take actions to mitigate it.

The impact of these differences in behaviour between MSM with and without anorectal swabs on prevalence of other diseases warrants further investigation, as using anorectal swabs as a marker of male-to-male sex may capture men at higher risk of STI acquisition. Men who did not have anorectal swabs were much more likely to be behaviourally bisexual; perhaps these men were less likely to engage in receptive anal intercourse, or were less open about their homosexual activity within the clinical consultation [14, 15]. Interestingly, men who had anorectal testing were statistically younger than those who did not. This may reflect true differences in sexual practices by age (for example, less receptive anal sex among older men) [16] or greater discomfort among older MSM to reveal their sexual practices. The latter is a potential concern, particularly in light of a US study which found that older men were more likely than younger men to have rectal chlamydia [17].

A small number of anorectal swabs (1\%) were conducted among non-self-identified MSM. This may reflect other sexual practices, for example anal-digital activity and sharing of sex toys between partners of either sex [9, 18], or misreporting of male-to-male sex. These cases account for a low and acceptable false-positive rate and are unlikely to impact surveillance and trend monitoring where anorectal swabbing is used to identify MSM.

This study had a number of limitations. First, the variables that could be included in the model were 
Table 4. Characteristics of men reporting MSM at time of test collection, by anorectallnon-rectal collection; development sample; per cent $(95 \%$ CI)*

\begin{tabular}{lllr}
\hline \hline Test collection episodes in MSM $(n=11002)$ & $\begin{array}{l}\text { Anorectal collection } \\
(n=9597)\end{array}$ & $\begin{array}{l}\text { Non-rectal collection } \\
(n=1405)\end{array}$ & $P$-value \\
\hline Median age, years & $29 \cdot 8(29 \cdot 6-30 \cdot 0)$ & $33 \cdot 8(32 \cdot 8-34 \cdot 7)$ & $<0 \cdot 001$ \\
Australian born $(n=10101)$ & $64 \cdot 5(63 \cdot 0-66 \cdot 1)$ & $62 \cdot 7(59 \cdot 5-65 \cdot 7)$ & $0 \cdot 252$ \\
$>10$ male partners in last $12 \mathrm{~m}$ & $30 \cdot 1(28 \cdot 9-31 \cdot 3)$ & $20 \cdot 6(18 \cdot 4-23 \cdot 1)$ & $<0 \cdot 001$ \\
Reported female partners in last $12 \mathrm{~m}(n=10852)$ & $9 \cdot 57(8 \cdot 84-10 \cdot 4)$ & $30 \cdot 9(28 \cdot 3-33 \cdot 7)$ & $<0 \cdot 001$ \\
Always use condom $\dagger(n=9994)$ & $46 \cdot 7(45 \cdot 4-47 \cdot 9)$ & $40 \cdot 8(37 \cdot 8-43 \cdot 8)$ & $<0 \cdot 001$ \\
Urogenital chlamydia positive at test collection $¥(n=10949)$ & $3 \cdot 07(2 \cdot 74-3 \cdot 44)$ & $3 \cdot 28(2 \cdot 46-4 \cdot 35)$ & $0 \cdot 678$ \\
\hline \hline
\end{tabular}

Total number of observations $(n)=11002$ for all comparisons, unless indicated.

* Cluster robust standard errors specified.

$\dagger$ Always used a condom during anal and vaginal sex in the previous 12 months.

$\$$ Refers to a chlamydia-positive urine or urethral test result at test collection episode.

primarily limited to variables regularly collected in surveillance systems in order to optimise applicability: i.e., age, sex, anatomical site of test. An exception was made to enable exclusions to reduce bias in our model (sex workers and HIV-positive men receiving chronic disease care), and these may not be replicable in other surveillance systems. For example, history of ever having an anorectal swab may be a better predictor of MSM status, but is unlikely to be available in surveillance systems without capability to track individuals over time. A subanalysis (data not shown) demonstrated limited additional predictive value of lifetime anorectal swab in this sample. Second, our models were developed using data from one site only. Low rates of anorectal testing outside of sexual health services may limit the utility of anorectal swabs as a surrogate for MSM in some surveillance systems $[19,20]$. The external validation of using anorectal swabbing as a marker of MSM in unrelated enhanced surveillance datasets, including those that represent the general community, to assess the performance of the model in diverse contexts is warranted. However, the lack of consistently collected behavioural data in most surveillance systems limits the options available for validation purposes. Finally, MSM status was based on a question asking about sexual contact with males but not specifying anal sex. MSM not engaging in receptive anal sex may decline an anorectal swab.

In conclusion, anorectal swabs are a valid marker of MSM behaviour in surveillance data from specialised sexual health centres. This marker provides a practical and sensitive means of identifying and following trends of STIs among MSM in surveillance systems where behavioural data are not routinely collected, thus extending the utility of laboratory and clinical surveillance data to monitor disease in a key population.

\section{ACKNOWLEDGEMENTS}

The authors gratefully acknowledge the patients and participating laboratories and clinics for providing data for the Victorian Primary Care Network for Sentinel Surveillance on Blood Borne Viruses and STIs (VPCNSS). The VPNSS is managed at the Burnet Institute and is in collaboration with the Victorian Infectious Diseases Reference Laboratory and Melbourne Sexual Health Centre. This work was supported by the Victorian Department of Health and Human Services. The VPCNSS has significant ongoing support from surveillance officers at the Burnet Institute. They gratefully acknowledge the contribution to this work of the Victorian Operational Infrastructure Support Program received by the Burnet Institute.

\section{REFERENCES}

1. Department of Health and Ageing. The Third National Sexually Transmissible Infections (STIS) Strategy 2014-2017. Canberra: Commonwealth of Australia, 2014 (http://www.health.gov.au/internet/main/publishing.nsf/Content/ohp-bbvs-sti)

2. Lowndes CM, Fenton KA. Surveillance systems for STIs in the European Union: facing a changing epidemiology. Sexually Transmitted Infections 2004; 80: 264 271. doi: 10.1136/sti.2004.010389.

3. Catchpole MA. The role of epidemiology and surveillance systems in the control of sexually transmitted diseases. Genitourinary Medicine 1996; 72: 321-329. 
4. Guy RJ, et al. A new national Chlamydia sentinel surveillance system in Australia: evaluation of the first stage of implementation. Communicable Diseases Intelligence Quarterly Report 2010; 34: 319-328.

5. Templeton DJ, et al. Australian sexually transmissible infection and HIV testing guidelines for asymptomatic men who have sex with men 2014: a review of the evidence. Sexual Health 2014; 11: 217-229. doi: 10.1071/ $\mathrm{SH} 14003$.

6. Sexually Transmissible Infections in Gay Men Action Group (STIGMA). Australian sexually transmissible infection \& HIV testing guidelines 2014 for asymptomatic men who have sex with men. Sydney: New South Wales STI Programs Unit, 2014. Available online at: http://www.stipu.nsw.gov.au/icms_docs/163932_STI_ testing_guidelines_for_MSM_2010.pdf.

7. World Health Organization. Guidelines: prevention and treatment of HIV and other sexually transmitted infections among men who have sex with men and transgender people: recommendations for a public health approach 2011. Geneva: World Health Organization, 2011.

8. Dukers-Muijrers NHTM, et al. What is needed to guide testing for anorectal and pharyngeal Chlamydia trachomatis and Neisseria gonorrhoeae in women and men? Evidence and opinion. BMC Infectious Diseases 2015; 15: 533. doi: 10.1186/s12879-015-1280-6.

9. van Liere GAFS, et al. Standard symptom- and sexual history-based testing misses anorectal Chlamydia trachomatis and Neisseria gonorrhoeae infections in swingers and men who have sex with men. Sexually Transmitted Infections 2013; 40: 285-289. doi: 10.1097/ OLQ.0b013e31828098f8.

10. Goller J, et al. Establishing a linked sentinel surveillance system for BBVs and STIs: methods, system attributes and early findings. Sexual Health 2010; 7: 425-433.

11. Kirby Institute. HIV, viral Hepatitis and Sexually Transmissible Infections in Australia Annual Surveillance Report 2015. Sydney: Kirby Institute, The University of New South Wales, 2015.
12. Hosmer DW, Lemeshow S, Sturdivant RX. Applied Logistic Regression, 3rd edn. New Jersey: John Wiley \& Sons, 2013.

13. StataCorp. Stata Statistical Software: Release $13 \cdot 1$. College Station, TX: StataCorp LP, 2013.

14. Bernstein KT, et al. Same-sex attraction disclosure to health care providers among New York City men who have sex with men: implications for HIV testing approaches. Archives of Internal Medicine 2008; 168: 1458-1464. doi: 10.1001/archinte.168.13.1458.

15. Friedman MR, et al. HIV infection and sexual risk among men who have sex with men and women (MSMW): a systematic review and meta-analysis. PLoS ONE 2014; 9: e87139. doi: 10.1371/journal.pone.0087139.

16. Rosenberger JG, et al. Sexual behaviors and situational characteristics of most recent male-partnered sexual event among gay and bisexually identified men in the United States. The Journal of Sexual Medicine 2011; 8: 3040-3050. http://dx.doi.org/10.1111/j.1743-6109.2011. 02438.x

17. Trebach JD, et al. Neisseria gonorrhoeae and Chlamydia trachomatis among women reporting extragenital exposures. Sexually Transmitted Infections 2015; 42: 233 239. doi: 10.1097/OLQ.0000000000000248.

18. van Liere GA, et al. High co-occurrence of anorectal chlamydia with urogenital chlamydia in women visiting an STI clinic revealed by routine universal testing in an observational study; a recommendation towards a better anorectal chlamydia control in women. BMC Infectious Diseases 2014; 14: 1-7. doi: 10.1186/1471-2334-14-274.

19. Dimech W, et al. Analysis of laboratory testing results collected in an enhanced chlamydia surveillance system in Australia, 2008-2010. BMC Infectious Diseases 2014; 14: 325. doi: 10.1186/1471-2334-14-325.

20. den Heijer CDJ, et al. Who tests whom? A comprehensive overview of Chlamydia trachomatis test practices in a Dutch region among different STI care providers for urogenital, anorectal and oropharyngeal sites in young people: a cross-sectional study. Sexually Transmitted Infections 2016; 92: 211-217. doi: 10.1136/sextrans-2015052065 . 